\title{
Synthesis and Properties for Benzotriazole Nitrogen Oxides (BTzO) and Tris[1,2,4]triazolo[1,3,5]triazine Derivatives
}

\author{
Yanyang Qu*, Qun Zeng, Jun Wang, Guijuan Fan, Jinglun Huang, Gaungchen Yang \\ Institute of Chemical Materials, China Academy of Engineering Physics, Mianyang, China \\ Email address: \\ quyy131226@caep.cn(Yanyang Qu) \\ ${ }^{*}$ Corresponding author \\ To cite this article: \\ Yanyang Qu, Qun Zeng, Jun Wang, Guijuan Fan, Jinglun Huang, Gaungchen Yang. Synthesis and Properties for Benzotriazole Nitrogen \\ Oxides (BTzO) and Tris[1,2,4]triazolo[1,3,5]triazine Derivatives. International Journal of Materials Science and Applications. \\ Vol. 7, No. 2, 2017, pp. 49-57. doi: 10.11648/j.ijmsa.20180702.13
}

Received: October 7, 2017; Accepted: October 23, 2017; Published: March 26, 2018

\begin{abstract}
Herein we first report a novel method of synthesis fused ring nitrogen-enriched compounds by intramolecular cyclization reaction. Some of them were characterized by IR, ${ }^{1} \mathrm{H}$ and ${ }^{13} \mathrm{C}$ NMR spectroscopy. Most of them exhibit outstanding positive heat of formation $(155-376 \mathrm{~kJ} / \mathrm{mol})$. Densities of these compounds fall in the range between 1.7321$1.8847 \mathrm{~g} . \mathrm{cm}^{-1}$, which places them in a class of relatively dense energetic materials. Their physical properties were evaluated by Gaussian 09, and EXPLO5 6.02 calculations. Their detonation velocities and pressures were calculated to fall in the range of 6713-8441 m.s ${ }^{-1}$ and 14.47-30.61 Gpa.
\end{abstract}

Keywords: Fused Heterocycles, 1,2,4-triazoles, tris[1,2,4]triazolo[1,3,5]triazine, 1,3,5-triazines, Benzotriazole Nitrogen Oxides, Synthetic Methods

\section{Introduction}

High-nitrogen heterocyclic systems have long been recognized as one class of useful and promising structures for the design and synthesis of high-energy compounds. [1] Of these, the 1, 2, 4-triazole, 1, 2, 3-triazole and triazine with a small ring tension, good stability, high nitrogen content, high enthalpy generation and easy to implement oxygen balance are efficient fragments to enhance the performance of highenergy compounds. Recently, the development of new energetic materials has drawn more attention on fused cyclic nitrogen-containing heterocycles. [2] Expecially, the combination of the 1, 2, 4-triazole or 1, 2, 3-trizole fragment with triazine and aromatic heterocyclic compounds would lead to new energetic performance which would possess positive properties of both classes.

Tris $[1,2,4]$ triazolo[1,3,5]triazine in which the triazine ring is fused to three triazole rings is designated as TTT. In the early 20 th century, the first tris $[1,2,4]$ triazolo[ $[1,3,5]$ triazine was prepared by heating 3,5-diamino-1,2,4-triazole (guanazole). [3] The assigned structure $\mathrm{Y}_{3} \mathrm{TTT}$ to this compound (Figure 1) and called it pyroguanazole. More recently, triphenyl substituted compound with the structure $\mathrm{X}_{3}$ TTT was prepared by Huisgen et al. [4] by the reaction of cyanuric chloride with phenyltetrazole.

To our knowledge, the above compounds are the only known TTT. This class of compounds is of interest from the standpoint of their biological activity and also because they are potential components of energetic materials with high thermal stability. The aim of this study is to develop new procedures for the synthesis of TTT and their derivatives. Meanwhile, we provide a facile methodology for synthesis of benzotriazole nitrogen oxides (BTzO). However, the properties about this type of fused ring nitrogen-enriched compounds has not been reported. 


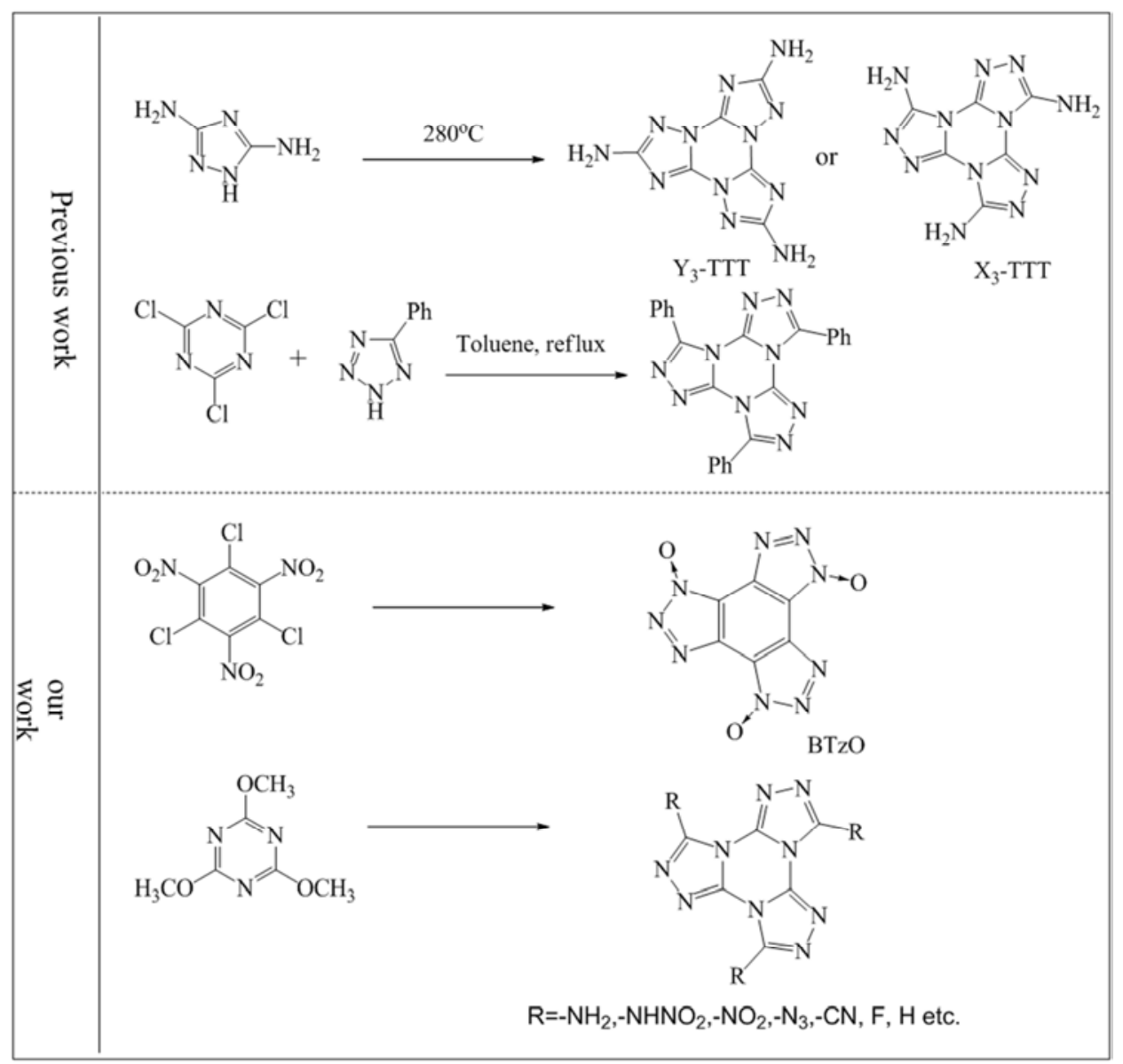

Figure 1. Example of placing the caption bellow the figure.

\section{Result and Discussion}

In this study, 3-(5-amino-1, 2, 4-triazole) [4, 3-a] [1, 3, 5] triazine (TATT) was synthesized using a modified route in which compound 1, 3, 5-triazine-2, 4, $6(1 \mathrm{H}, 3 \mathrm{H}, 5 \mathrm{H})$-trione trihydrazone was prepared by one-pot reaction starting with 2,4,6-trimethoxy-1,3,5-triazine in yields approaching 97\%. The product was sufficiently pure to be used directly in the following reactions. With 2,4,6-trihydrazine-1,3,5-triazine in hand, the fused tris $[1,2,4]$ triazolo $[1,3,5]$ triazine ring was easily constructed through cyclization reaction using cyanogen bromide. (As shown in Figure 2). In the process of the reaction, brown solid will be precipitated, which directly to the suction filter, washing and drying can be easy to get the required products in $52 \%$ yields.<smiles>COc1nc(OC)nc(OC)n1</smiles>

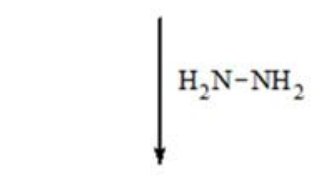<smiles>NNc1nc(NN)nc(NN)n1</smiles><smiles>Cc1nnc2n1c1nnc(N)n1c1nnc(N)n21</smiles>

TATT

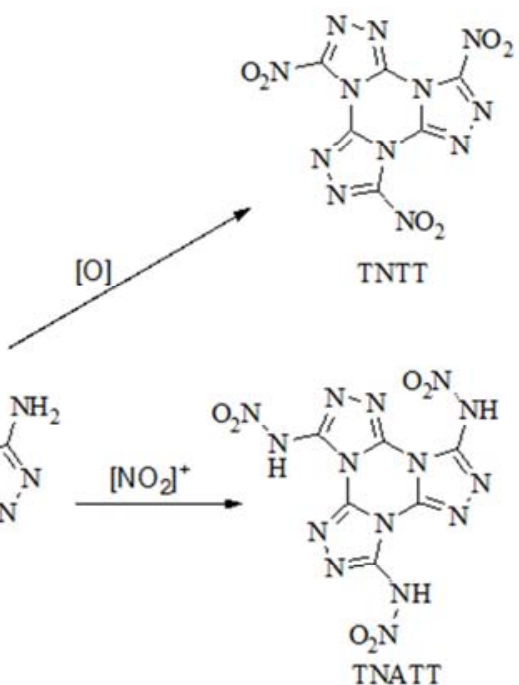

Figure 2. Synthesis of TATT and TNATT. 
The determination of ESI-MS spectrum of synthetic products by adopting the positive ion mode. As shown in Figure 3. TATT quasi molecular ion peak $[\mathrm{M}+\mathrm{H}]^{+} 246.9$ is clearly visible. Specifies that the molecular weight of the synthetic compounds is correct. The TATT was further characterized by ${ }^{1} \mathrm{H}$ and ${ }^{13} \mathrm{C}$ NMR spectrum respectively as shown in Figure 4 and Figure 5. In the ${ }^{1} \mathrm{H}$ NMR, in addition to water and solvent peak, only an amino proton signals, and ${ }^{13} \mathrm{C}$ NMR, besides the solvent peak, only two kinds of carbon signal. Therefore, from the point of nuclear magnetic spectra, the structure can be identified with good symmetry as 3-(5amino-1, 2, 4-triazole) [4, 3-a] [1, 3, 5] triazine (TATT). Furhter, the TATT structure was characterized by IR spectroscopy. (As shown in Figure 6).

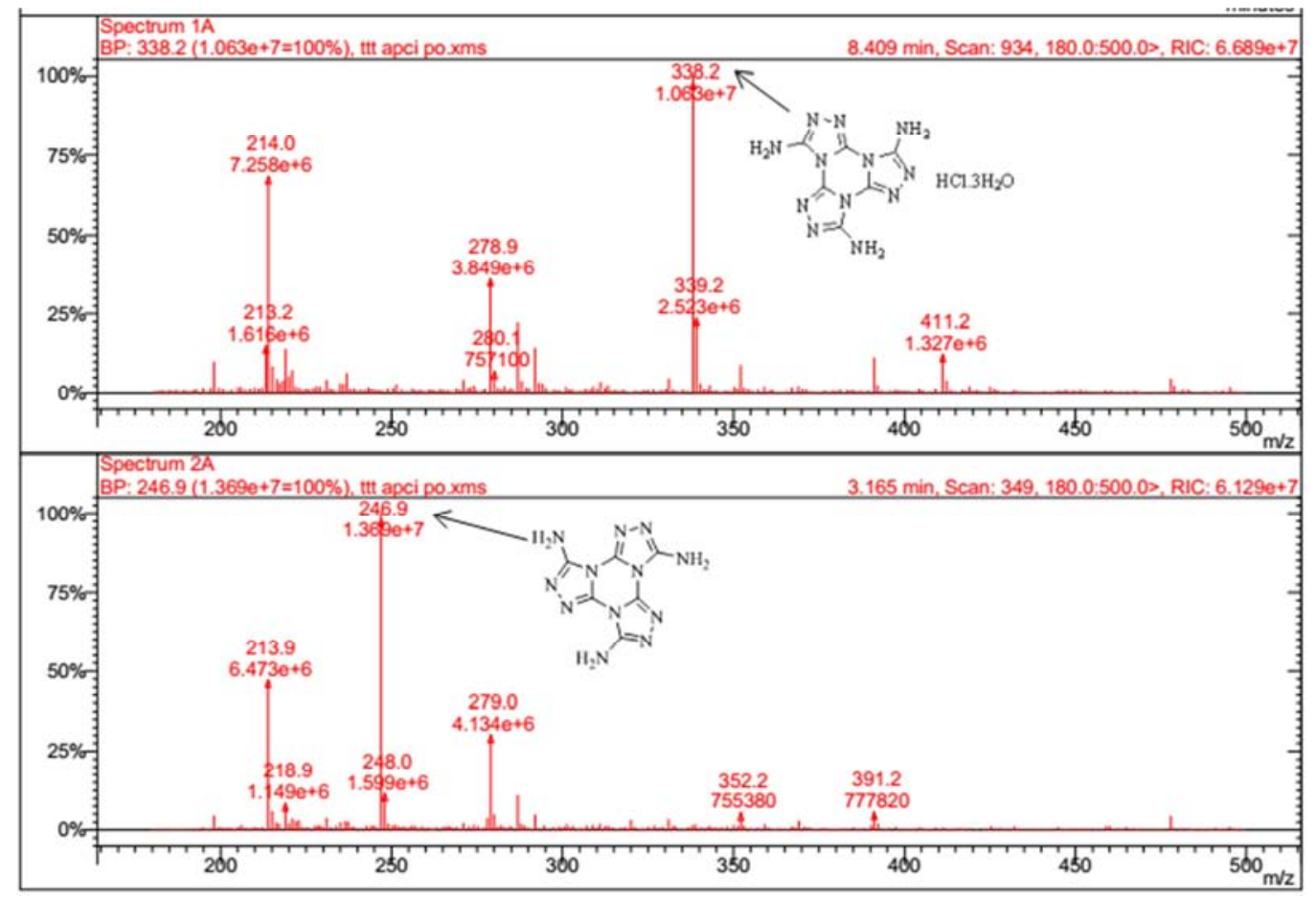

Figure 3. ESI-MS spectrum of TATT.

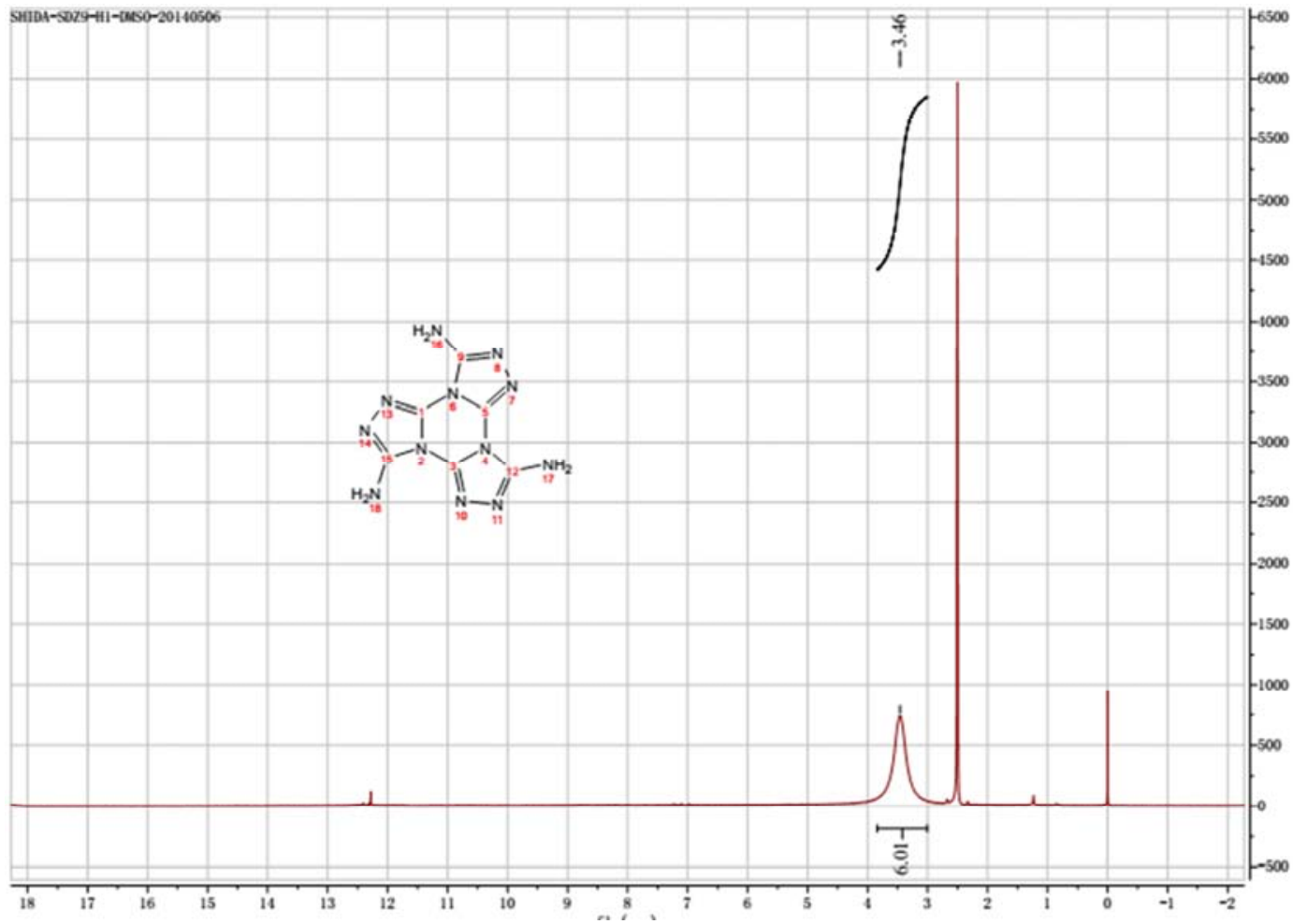

Figure 4. ${ }^{1} H$-NMR spectrum of TATT. 


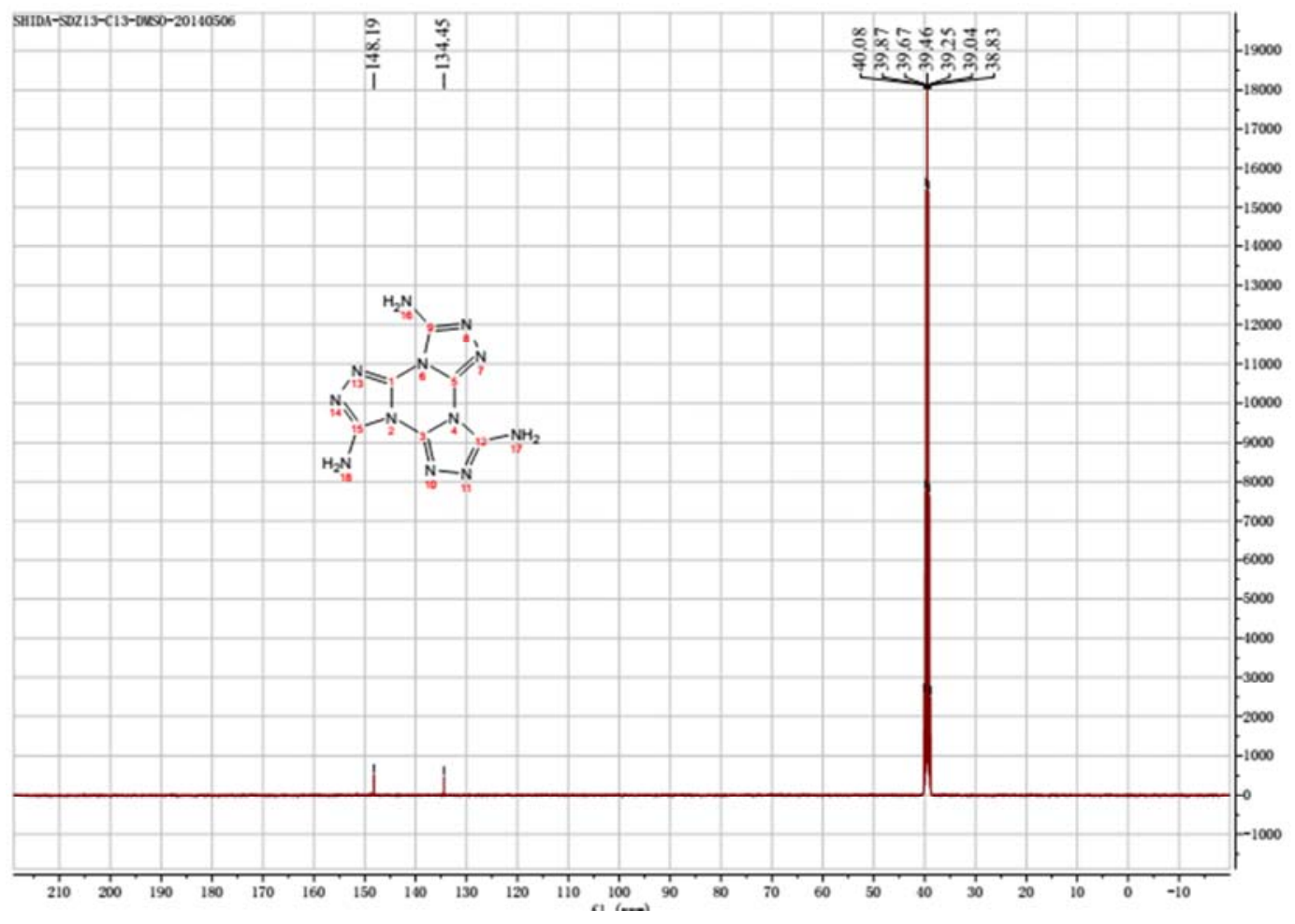

Figure 5. ${ }^{13} \mathrm{C}$-NMR spectrum of TATT.

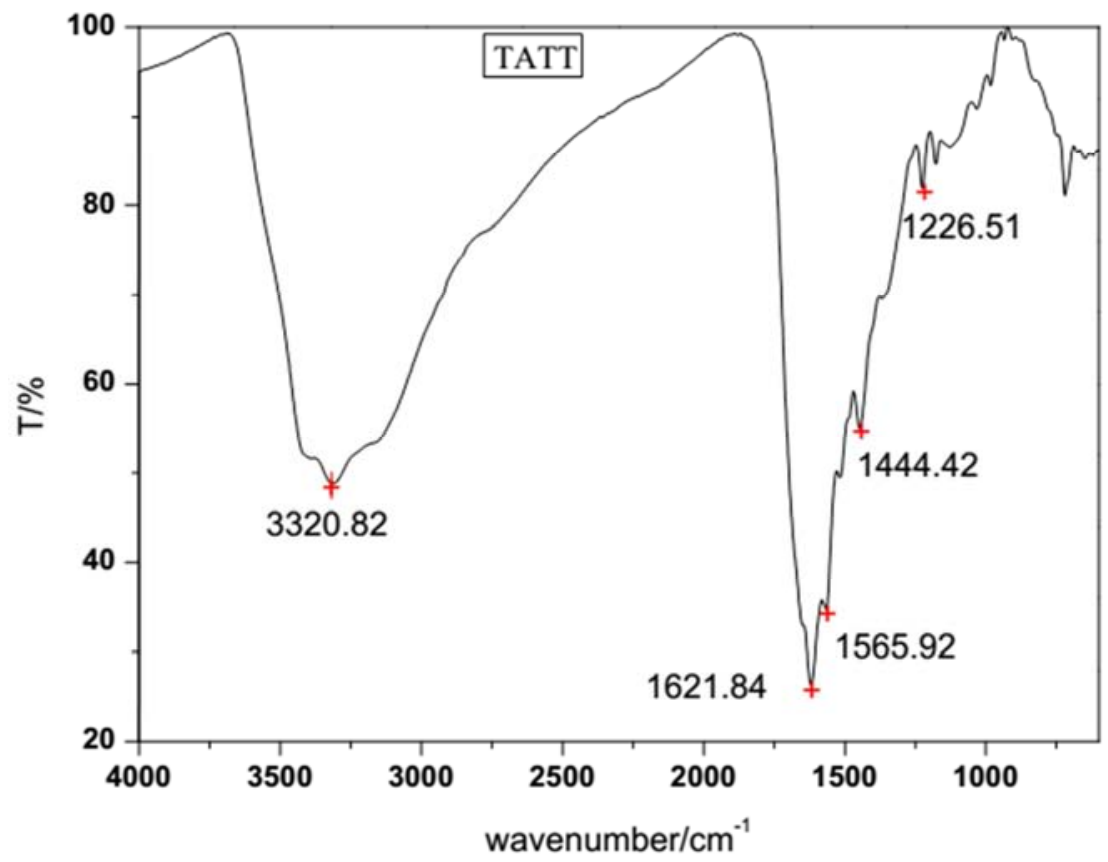

Figure 6. IR spectrum of TATT.

Several reagents for the nitration of the TATT ring are available. Here mixture of fuming nitric acid and acetic anhydride and nitration reagent $\mathrm{NO}_{2} \mathrm{BF}_{4}$ were used for the nitration of TATT to get 3-amino-1 (5-nitrate-4trichlorobenzene triazole) $[4,3-\mathrm{a}][3,5,1]$ triazine (TNATT) in yields between $17 \%$ and $32 \%$, respectively. (As shown in Table 1). Through the ESI - MS to determine the molecular weight of the product is correct (Figure. 7). Because of the poor solubility, we unable to find suitable deuterated solvent, thus did not measure its NMR spectra.

Table 1. The nitrate amination reaction of TATT.

\begin{tabular}{llll}
\hline Entry & nitration reagent & reaction condation & TNATT (yield\%) \\
\hline 1 & $\mathrm{NO}_{2} \mathrm{BF}_{4}$ & $-20^{\circ} \mathrm{C}-10^{\circ} \mathrm{C} / 12 \mathrm{~h}$ & 27 \\
2 & $\mathrm{NO}_{2} \mathrm{BF}_{4}$ & $-30^{\circ} \mathrm{C}-10^{\circ} \mathrm{C} / 12 \mathrm{~h}$ & 22 \\
3 & $\mathrm{NO}_{2} \mathrm{BF}_{4}$ & $-30^{\circ} \mathrm{C}-10^{\circ} \mathrm{C} / 6 \mathrm{~h}$ & 19 \\
4 & $95 \% \mathrm{HNO}_{3}-\mathrm{Ac}_{2} \mathrm{O}$ & $-20^{\circ} \mathrm{C}-0^{\circ} \mathrm{C} / 12 \mathrm{~h}$ & 32 \\
5 & $95 \% \mathrm{HNO}_{3}-\mathrm{Ac}_{2} \mathrm{O}$ & $2^{\circ} \mathrm{C} / 12 \mathrm{~h}$ & 17 \\
\hline
\end{tabular}




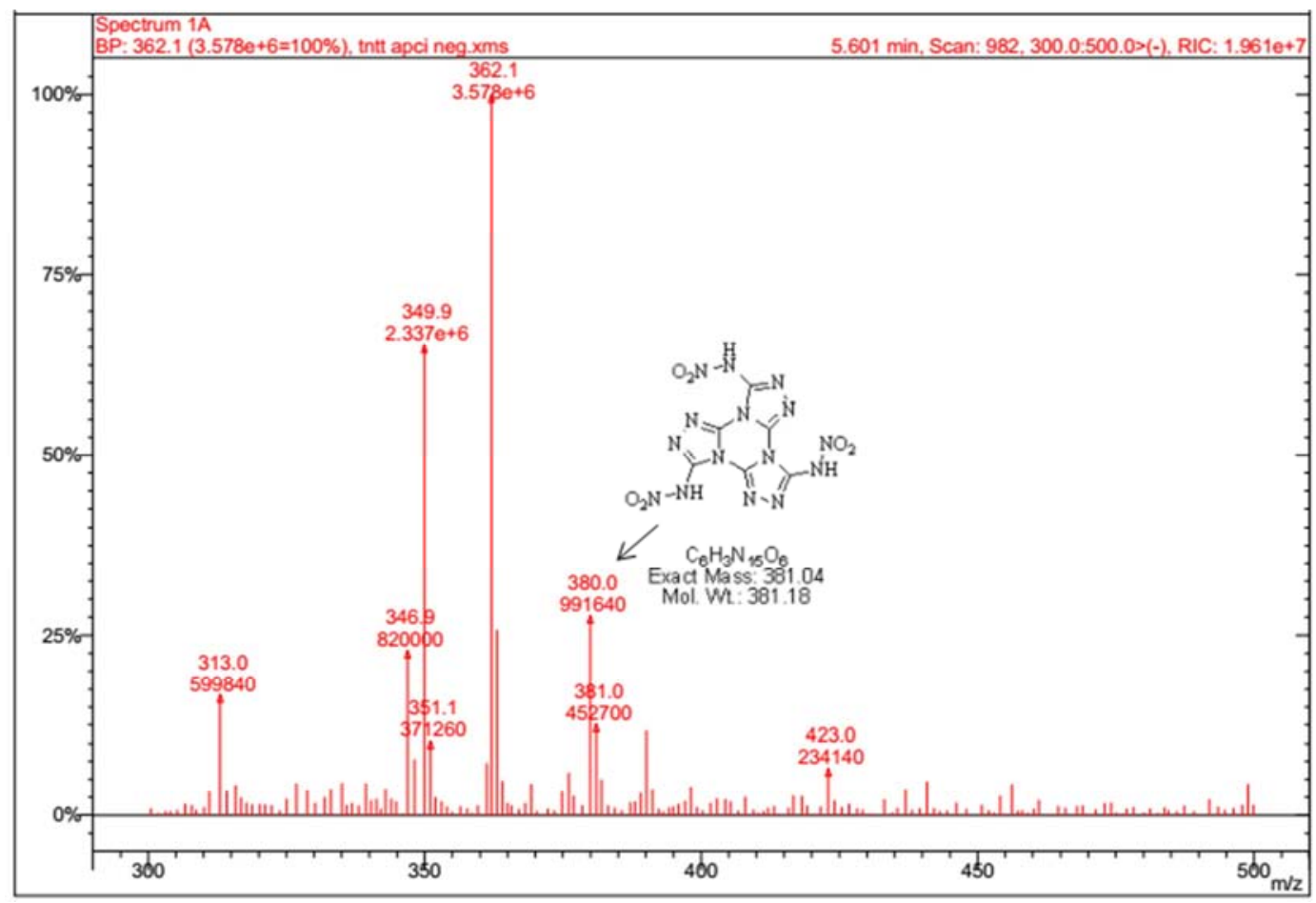

Figure 7. ESI-MS spectrum of TNATT.

In order to prepare 3-(5-nitro-1, 2, 4-triazole) [4, 3-a] [1, 3, 5] triazine (TNTT) energetic compound, we tried a variety of oxidizing reagents, such as: $\mathrm{H}_{2} \mathrm{O}_{2}-\mathrm{H}_{2} \mathrm{SO}_{4}$, with $\mathrm{Na}_{2} \mathrm{WO}_{4}$, $\mathrm{H}_{2} \mathrm{O}_{2} / \mathrm{H}_{2} \mathrm{SO}_{4}$, fuming $\mathrm{HNO}_{3}, \mathrm{NaNO}_{2} / \mathrm{H}_{2} \mathrm{SO}_{4}, 100 \%$
$\mathrm{HNO}_{3} / \mathrm{CH}_{2} \mathrm{Cl}_{2}, \mathrm{H}_{2} \mathrm{O}_{2} /\left(\mathrm{NH}_{4}\right)_{2} \mathrm{~S}_{2} \mathrm{O}_{8} / \mathrm{H}_{2} \mathrm{SO}_{4}$, $\mathrm{H}_{2} \mathrm{O}_{2} / \mathrm{CH}_{3} \mathrm{CN} / \mathrm{H}_{2} \mathrm{SO}_{4}, \mathrm{KNO}_{3} / \mathrm{H}_{2} \mathrm{SO}_{4}, \mathrm{H}_{2} \mathrm{O}_{2} / \mathrm{CH}_{3} \mathrm{SO}_{3} \mathrm{H}$ and $\mathrm{H}_{2} \mathrm{O}_{2} /\left(\mathrm{CF}_{3} \mathrm{CO}\right)_{2} \mathrm{O}$ using a procedure similar to that described in the literature. [5] (As shown in Figure 8).
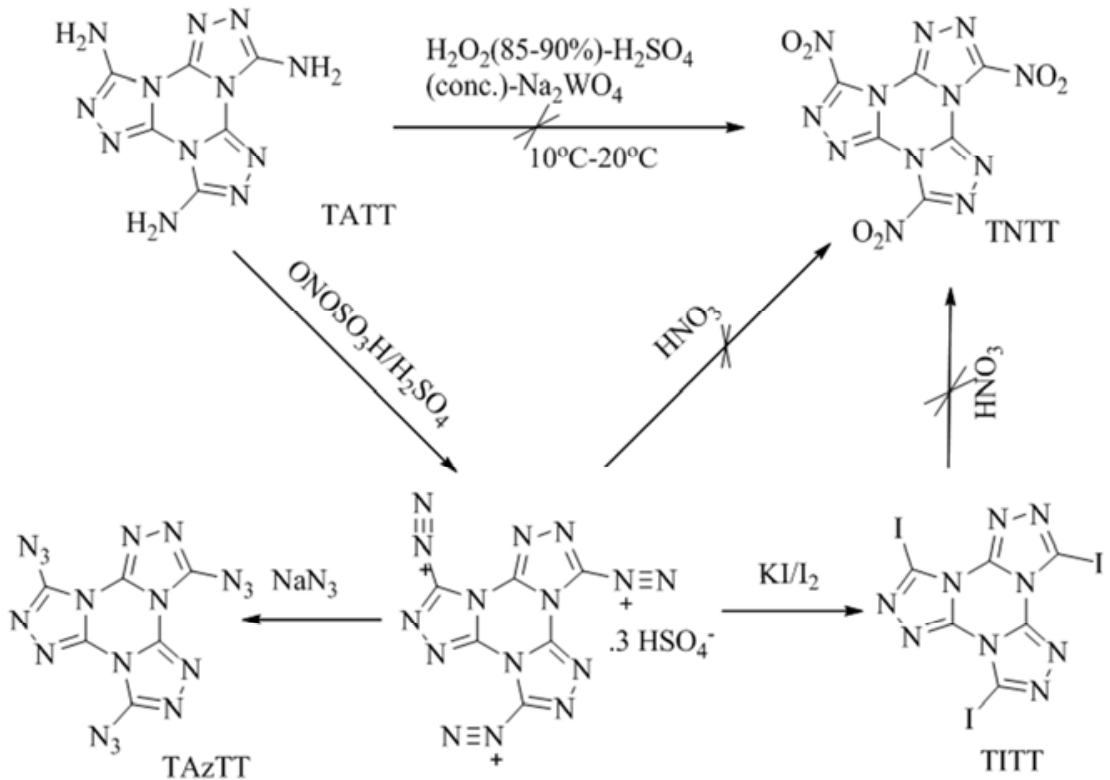

Figure 8. Reactions of TATT with different reagents.

Unfortunately, the compounds TNTT did not result when these method were used. Most of the reactions raw materials can be completely disappear, when pour into the ice water, we won't get any solids, and some reactions can be precipitated a small amount of yellow or black solid product, but did not detect target product molecular weight and its intermediates by MS spectra. Which may be due to consider two reasons, one of the reasons is the molecular symmetry is very good, no $\mathrm{H}$ atoms in the structure, and it is difficult to ionization under the condition of the mass spectrum of being used. Another reason is that there are bridge a nitrogen atom in the target molecule and the structure is not stable, therefore, 
in the reaction conditions have side effects, such as broken key reaction, thus no solid precipitation.

By analogy with the similar method,[6] we attempted to synthesized 3-benzo[(1, 2, 3) - triazole-N-oxide] (BTzO) by reacting trichlorotrinitrobenzene (TCTNB) with hydrazine hydrate in ethonal at room temperature. However, this attempt was unsuccessful. Heating to $60^{\circ} \mathrm{C}$ in ethonal afforded compound $\mathrm{BTzO}$ in lower yield $(12 \%)$. The best results were obtained upon with sodium bicarbonate solution, after 5 hours the reaction to $60^{\circ} \mathrm{C}$ and continue to reaction for 36 hours (the yield of $\mathrm{BTzO}$ was $46 \%$ ). Compound $\mathrm{BTzO}$ begins to decompose only at about $230^{\circ} \mathrm{C}$. For thermolysis of TCTNB to form $\mathrm{BTzO}$ structure, it is necessary that the $\mathrm{C}$ atom of benzene ring be the attacking nucleophile in the first steps of the reaction, and the $\mathrm{N}$ atom of the intermediate linear trimer be the attacking nucleophile in the second step. (As shown in Figure 9).

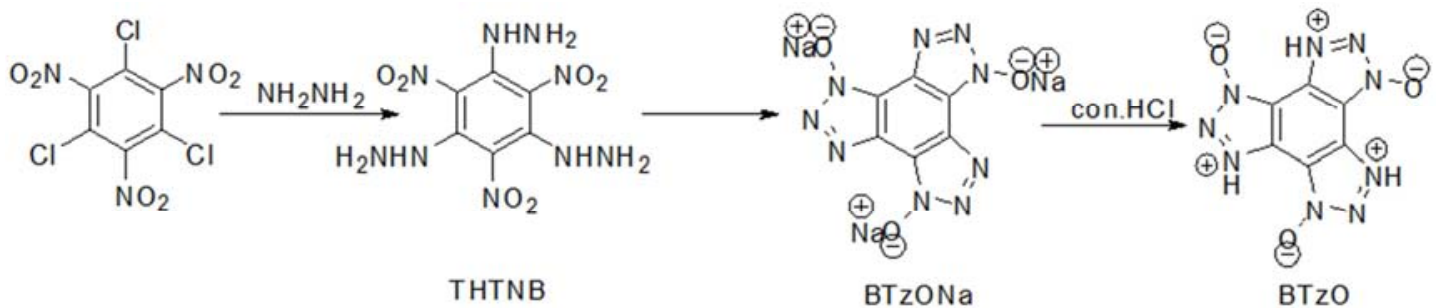

Figure 9. Synthesis of 3-benzo [(1, 2, 3)-triazole-N-oxide].

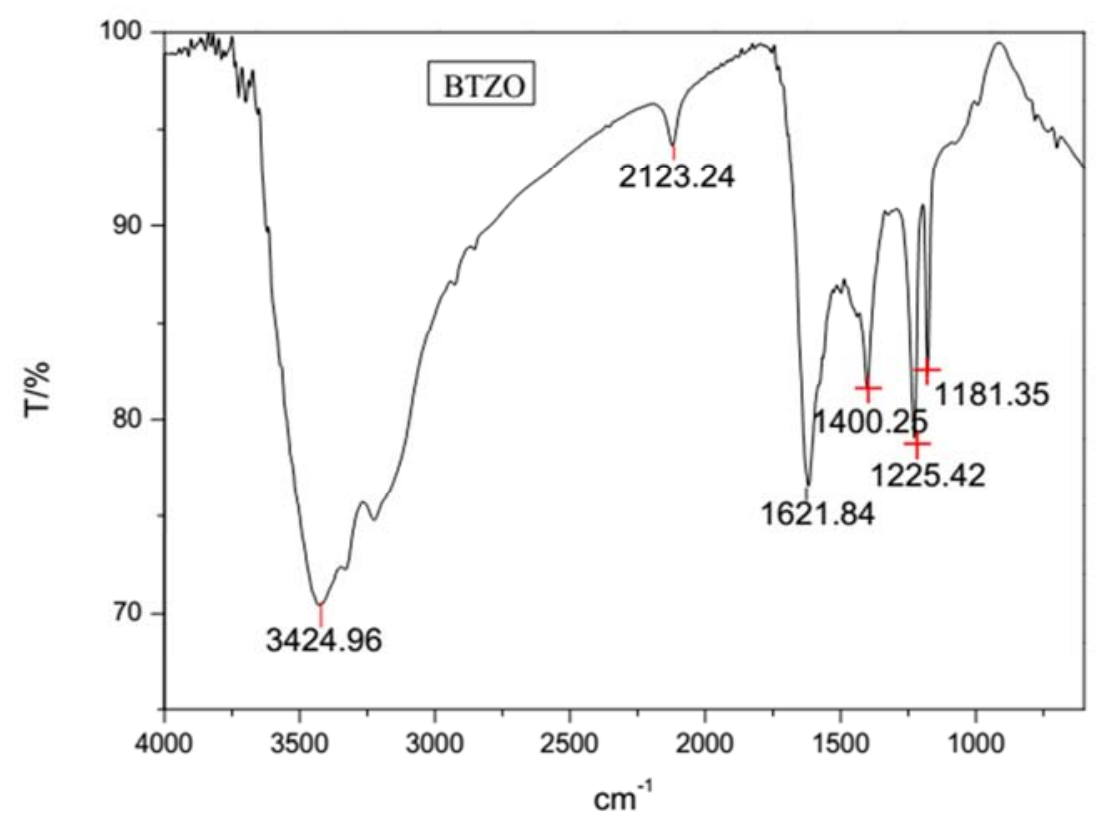

Figure 10. IR spectrum of $B T z O$.

\section{Thermal Stability of BTzO}

According to the DSC experiments we tested thermal decomposition of the data with $2.5 \mathrm{~K} \cdot \mathrm{min}^{-1}, 5 \mathrm{~K} \cdot \mathrm{min}^{-1}, 10$ $\mathrm{K} \cdot \mathrm{min}^{-1}, 20 \mathrm{~K} \cdot \mathrm{min}^{-1}$ four different heating rate and the results as shown in Figure 11. Only exothermic peak four DSC curves, and almost can't see the endothermic melting peak. This illustrates the $\mathrm{BTzO}$ solid decomposition and molten decomposition show the same trend.

Using Kissinger and Ozawa method to calculate the thermal decomposition apparent active energy E, with the related coefficient as shown in equation (1) and (2). The calculate resultshows the correlation of two kinds calculate method is better, the activation energy is very close, which explain the $\mathrm{BTzO}$ had good thermal stability. (As shown in table 2).

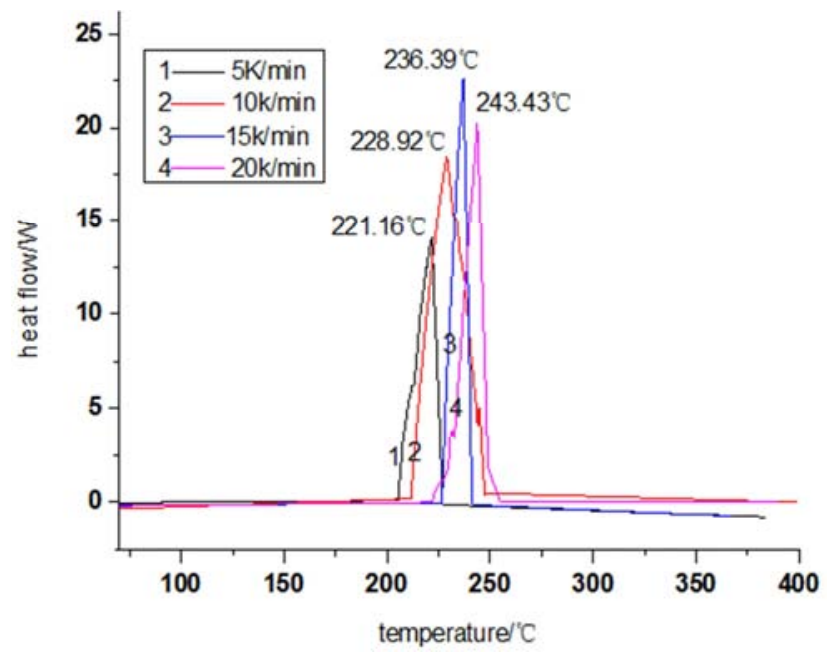

Figure 11. DSC diagram of $B T z O$. 


$$
\begin{gathered}
\ln \left(\frac{\beta}{T_{p}^{2}}\right)=\ln \left(\frac{A R}{E}\right)-\frac{E}{R T_{p}} \\
\lg \beta=\lg \frac{A E}{\operatorname{Rg}(\alpha)}-2.315-0.4567 \frac{E}{R T_{p}}
\end{gathered}
$$

$\beta$ : heating rate, $\mathrm{K} / \mathrm{min}$; Tp: peak temperature, $\mathrm{K}$; $\mathrm{R}$ : the ideal gas constant, $\mathrm{J} /(\mathrm{K} \cdot \mathrm{min})$; $\mathrm{A}$ : former factor, $\mathrm{s}^{-1}$; $\mathrm{E}$ : apparent activation energy, $\mathrm{kJ} / \mathrm{mol} ; \mathrm{g}(\alpha)$ : the function of thermal decomposition mechanism.

\begin{tabular}{|c|c|c|c|c|c|c|}
\hline \multirow{2}{*}{ Samples } & \multicolumn{3}{|c|}{ Kissinger's method } & \multicolumn{2}{|c|}{ Ozawa's method } & \multirow[t]{2}{*}{$\overline{\mathbf{E}}(\mathrm{kJ} / \mathrm{mol})$} \\
\hline & $\mathrm{E}(\mathrm{kJ} / \mathrm{mol})$ & $\ln A\left(s^{-1}\right)$ & $\mathbf{R}^{2}$ & $\mathrm{E}(\mathrm{kJ} / \mathrm{mol})$ & $\mathbf{R}^{2}$ & \\
\hline $\mathrm{BTzO}$ & 126.75 & 29.74 & 0.9534 & 124.78 & 0.9592 & 128.43 \\
\hline
\end{tabular}

Table 2. The apparent kinetic parameters of BTZO.

\section{Physical and Energetic Properties}

Density is one of the most attention performance factors for energetic materials. To obtain the density $(\rho)$, empirical equation (3) was chosen to handle the problem just depending on molecule directly. Although the regular flat structures should have good potential to packing with $\pi-\pi$ style, these compounds with TTT framework do not exhibit a much higher density than $2.044 \mathrm{~g} / \mathrm{cm}^{3}$ of $\varepsilon$-CL-20. Among them, compounds TNTT with nitro groups have the largest densities with $1.8847 \mathrm{~g} / \mathrm{cm}^{3}$, very close to $1.879 \mathrm{~g} / \mathrm{cm}^{3}$ of BTF in calculation. Compound TNATT with nitroamine group is followed with the density of 1.866 $\mathrm{g} / \mathrm{cm}^{3}$. And compound TAzTT with azido group have the density of $1.803 \mathrm{~g} / \mathrm{cm}^{3}$. However, For compound $\mathrm{BTzO}$, the density of is estimated to be $1.873 \mathrm{~g} / \mathrm{cm}^{3}$.

The heat of formation were evaluated with a isodesmis reaction methods at G3B3 level with Gaussian 09 A.02 for all compounds. The condensed-phase $\operatorname{HOF}\left(\Delta_{f} H_{\text {Cond }}^{\circ}\right)$ were obtained combining with $\Delta H_{\text {sub }}$ predicted using Eq (4) and (5). The HOF of BTF was evaluated to be $597.8 \mathrm{~kJ} / \mathrm{mol}$, very close to the experimental measure of $601.7 \mathrm{~kJ} / \mathrm{mol}$. [7]

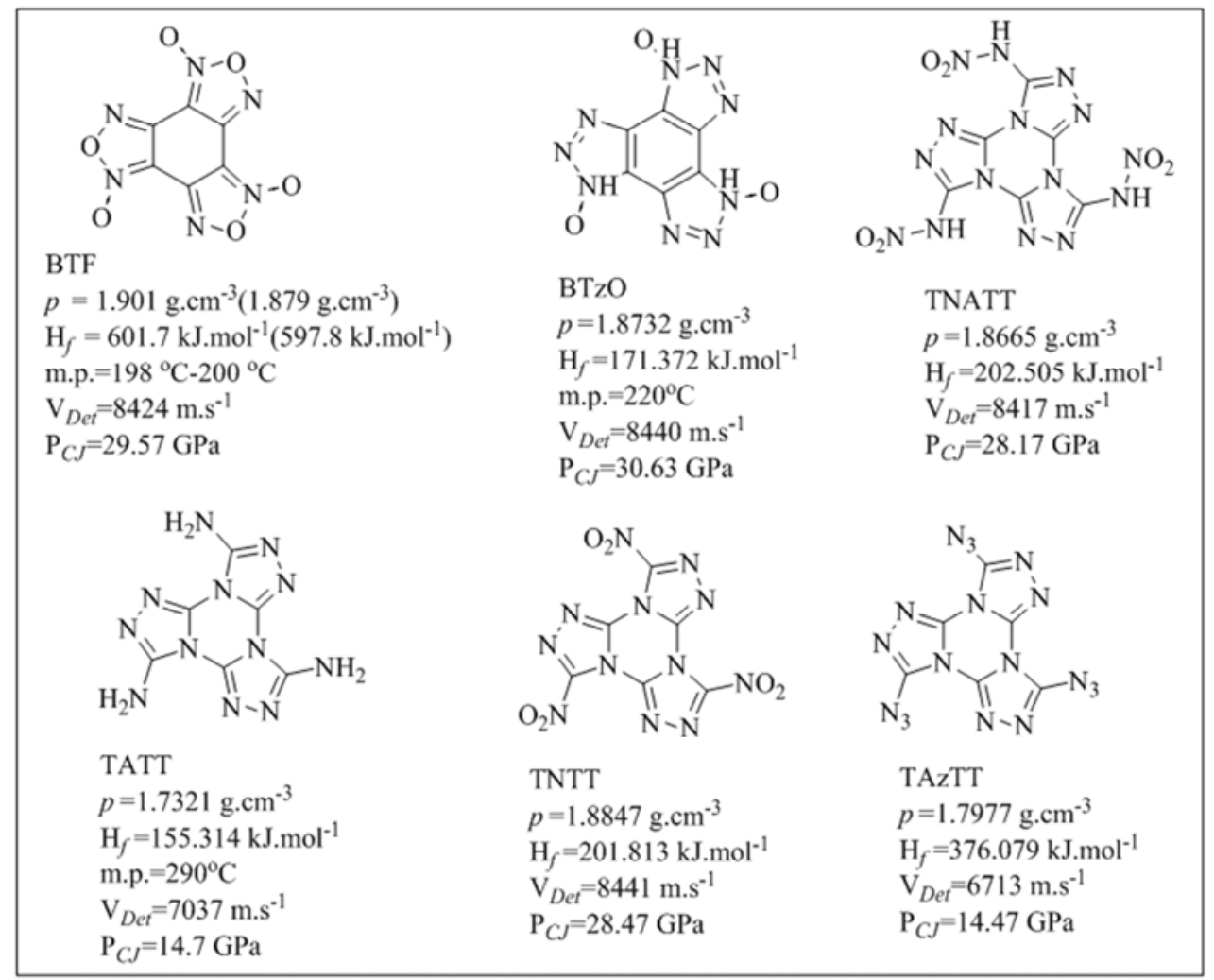

Figure 12. Thermodynamic properties of some compounds.

Together with the predicted crystal density and $\Delta_{f} H_{\text {Cond }}^{\circ}$, the detonation velocity (D) and pressure (P) were evaluated by EXPLO5 6.02. Figure 12 summarizes the detonation properties of these compounds as well as BTF. It's found that at the density of $1.879 \mathrm{~g} / \mathrm{cm}^{3}$, the $D$ and $P$ of BTF were predicted to be $8424 \mathrm{~m} \cdot \mathrm{s}^{-1}$ and $29.57 \mathrm{Gpa}$. In these compounds, TNTT and $\mathrm{BTzO}$ represent great performances $\left(D=8441 \mathrm{~m} \cdot \mathrm{s}^{-1}, \mathrm{P}=28.47 \mathrm{GPa}\right.$ and $D=8440 \mathrm{~m} \cdot \mathrm{s}^{-1}, \mathrm{P}=$ $30.63 \mathrm{GPa}$, respectively), which very close to the corresponding values of BTF. Moreover, compound TNATT and TAzTT also exhibit good detonation performances. 


\section{Calculation Method}

More detailed about theoretical studies on the derivatives of benzotriazole nitrogen oxides (BTzO) and tris $[1,2,4]$ triazolo $[1,3,5]$ triazine derivatives have been developed in our group. [8]

The density of energetic materials estimated with a empirical expression. [9]

$$
\rho=\beta_{1}\left(\frac{M}{V}\right)+\beta_{2}\left(v \sigma_{t o t}^{2}\right)+\beta_{3}
$$

In this study, a series of isodesmic reactions were design to obtain the standard gas-phase HOFs $\left(\Delta_{f} H_{\text {gas }}^{\circ}\right)$ at $298 \mathrm{~K}$, as shown follow:

For each isodesimic reaction, the $\Delta_{f} H_{\text {gas, 298K }}^{\circ}$ can be obtained from the following equation:

$$
\Delta_{f} H_{\mathrm{gas}, 298 \mathrm{~K}}^{\circ}=\sum \Delta_{f} H_{\mathrm{P}}-\sum \Delta_{f} H_{\mathrm{R}}=\Delta E_{0}-\Delta \mathrm{ZPE}+\Delta H_{\mathrm{T}}+\Delta n R T
$$

where $\Delta_{f} H_{\mathrm{P}}$ and $\Delta_{f} H_{\mathrm{R}}$ are the HOFs of the products and reactants at $298 \mathrm{~K}$, respectively. $\Delta E_{0}$ is the difference between the electron energy of the products and reactants at 0 $\mathrm{K}, \triangle \mathrm{ZPE}$ is the difference between the zero-point energy (ZPE) of the products and reactants, and $\Delta H_{\mathrm{T}}$ is the thermal correction form $0 \mathrm{~K}$ to $298 \mathrm{~K} . \Delta n R T$ is work term, which equals zero.

Based on Hess's law,[9] the condensed-phase HOFs $\left(\Delta_{f} H_{\text {Cond }}^{\circ}\right)$ were obtained by

$$
\Delta_{f} H_{\mathrm{Cond}}^{\circ}=\Delta_{f} H_{\mathrm{gas}}^{\circ}-\Delta H_{\mathrm{sub}}
$$

where $\Delta H_{\text {sub }}$ is the heat of sublimation and can be evaluated by the Byrd and Rice method[10] in the framework of the Politzer approach, [11] which has been successfully applied to many energetic compounds, using the following empirical expression:

$$
\Delta H_{\mathrm{sub}}=\alpha_{1} A^{2}+\alpha_{2}\left(v \sigma_{\mathrm{tot}}^{2}\right)^{0.5}+\alpha_{3}
$$

$\mathrm{A}$ and $\mathrm{V}$ are the area of isosurface of 0.001 electrons $/ \mathrm{bohr}^{3}$, and the volume contained within such isosurface, respectively; $v$ is the degree of balance between positive and negative potential on the molecular surface, $\sigma_{\text {tot }}^{2}$ is a measure of variability of total electrostatic potential. $\alpha_{i}$ and $\beta_{i}$ $(i=1,2,3)$ is empirical parameters fitting from experiment measure. $[10,12]$ Here, all of these terms about surface are obtained with Multiwfn program ${ }^{35}$ using wave functions from M06-2X/cc-pVTZ.

\section{Conclusion}

In summary, $\mathrm{BTzO}$ and TATT derivatives have been recommend for fused ring nitrogen-enriched energetic compounds, which were prepared using intramolecular cyclization method and some of them were characterized by IR,
${ }^{1} \mathrm{H}$ and ${ }^{13} \mathrm{C}$ NMR spectroscopy. Most of them exhibit outstanding positive heat of formation (155-376 kJ/mol). Densities of these compounds fall in the range between 1.73211.8847 g.cm ${ }^{-1}$, which places them in a class of relatively dense energetic materials. Their physical properties were evaluated by Gaussian 09. and EXPLO5 6.02 calculations. Their detonation velocities and pressures were calculated to fall in the range of 6713-8441 m.s ${ }^{-1}$ and 14.47-30.61 Gpa. Finally, all of compounds show highiy heat of formation, high percentage of nitrogen content with relatively good energetic properties. The results show compound $\mathrm{BTzO}$, TNTT and TNATT have the potential to release great power like BTF. As potential explosives, these compounds are worthy to pay more attention. These results are expected to provide some meaning information for people interested in these compounds.

\section{Acknowledgements}

The authors gratefully acknowledge the support of National Natural Science Foundation of China (NO. 11402240) and Foundation of CAEP (No. 2014B0302038). We are indebted to and thank Qianhong $\mathrm{Yi}$ and $\mathrm{Yu}$ Chi for considerable assistance with DSC test.

\section{References}

[1] a) H. X. Gao and J. M. Shreeve, Chem. Rev. 2011, 111 (11), 7377-7436; b) J. H. Zhang, C. L. He, D. A. Parrish and J. M. Shreeve, Chem.-Eur. J., 2013, 19 (27), 8929-8936; c) P. Yin, D. A. Parrish and J. M. Shreeve, J. Am. Chem. Soc., 2015, 137, 4778-4786; d) A. Hammerl, T. M. Klapotke, R. Rocha, Eur J Inorg Chem, 2006, 16, 2210-2228; f) T. M. Klapötke, M. Q. Kurz, P. C. Schmid and J. Stierstorfer, J. Energ. Mater., 2015, 33, 191-201; g) P. Yin, J. M. Shreeve, Advances in Heterocyclic Chemistry, 2017, 121, 89-131.

[2] Choose some of examples: a) G. K. Khisamutdinov, V. L. Korolev, T. N. Parkhomenko, V. M. Sharonova, E. S. Artemeva, I. S. Abdrakhmanov, S. P. Smirnov and B. I. Ugrak, Russ. Chem. Bull., 1993, 42 (10), 1700-1702; b) A. B. Sheremetev and I. L. Yudin, Usp. Khim., 2003, 72 (1), 93-107; c) Y. C. Li, C. Qi, S. H. Li, H. J. Zhang, C. H. Sun, Y. Z. Yu and S. P. Pang, J. Am. Chem. Soc., 2010, 132 (35), 12172$12173 ;$ d) A. A. Dippold and T. M. Klapötke, J. Am. Chem. Soc., 2013, 135 (26), 9931-9938; e) L. Vereshchagin, O. N. Verkhozina, F. A. Pokatilov, et al. Chemistry of Heterocyclic Compounds, 2010, 46, 206-211; f) V. Kizhnyaev, F. Pokatilov, L. Vereshchagin, et al. Russian Journal of Applied Chemistry, 2009, 82, 1769-1775; g) M. C. Schulze, B. L. Scott and D. E. Chavez, J. Mater. Chem. A, 2015, 3, 1796317965.

[3] a) K. A. Hofmann and O. Erhart, Chem. Ber., 1912, 45, 27312740; b) D. W. Kaiser, G. A. Peters, and V. P. Wystrach, J. Org. Chem., 1953, 18, 1610-1615; c) V. A. Tartakovsky, A. E. Frumkin, A. M. Churakov, and Yu. A. Strelenko, Russian Chemical Bulletin, International Edition, 2005, 54, 3, 719725; d) C. J. Snyder, T. W. Myers, D. E. Chavez, J. M. Veauthier, and R. J. Scharff, New Trends in Research of Energetic Materials, Czech Republic, 2016, 974-978.

[4] R. Huisgen, H. V. Sturm, and M. Seidel, Chem. Ber., 1961, 94, $1555-1562$. 
[5] a) A. K. Zelenin, M. L. Trudell, J. Heterocycle Chem. 1998, 35, 151-154; b) L. V. Batog, L. S. Konstantinova, and V. Yu. Rozhko, Russian Chemical Bulletin, International Edition, 2005, 54, 8, 1915-1922.

[6] a) C. L. He, J. H. Zhang, D. A. Parrish and J. M. Shreeve, J. Mater. Chem. A. 2013, 1, 2863-2868; b) T. M. Klapötke, C. Pflüger, M. Snćeska, New Trands in Research of Energetic Materials, Czech Republic, 2014, 754-768.

[7] a) P. J. Linstrom and W. G. Mallard, NIST Chemistry WebBook. (NIST, Gaithersburg, MD, 2005); R. L. Simpson, P A. Urtiew, D. L. Ornellas, G. L. Moody, K. F. J. Scribner and D. M. Hoffman, Propellants Explosives Pyrotechnics, 1997, 22 (5), 249-255; c) Q. Wu, W. H. Zhu and H. M. Xiao, RSC $A d v .$, 2014, 4 (8), 3789-3797; d) Q. H. Zhang, J. H. Zhang, X. J. Qi and J. M. Shreeve, J. Phys. Chem. A., 2014, 118 (45), 10857-10865.
[8] a) Q. Zeng, Y. Y. Qu, J. S. Li, H. Huang, $R S C A d v$., 2016, 6, 5419-5427; b) Q. Zeng, Y. Y. Qu, J. S. Li and H. Huang, RSC Adv., 2016, 6, 77005-77012.

[9] a) P. Politzer, J. Martinez, J. S. Murray and M. C. Concha, Mol. Phys., 2010, 108 (10), 1391-1396; b) B. M. Rice and E. F. C. Byrd, J. Comput. Chem., 2013, 34 (25), 2146-2151.

[10] E. F. C. Byrd and B. M. Rice, J. Phys. Chem. A., 2006, 110 (3), 1005-1013.

[11] P. Politzer, J. S. Murray, M. E. Grice, M. Desalvo and E. Miller, Mol. Phys., 1997, 91 (5), 923-928.

[12] a) B. M. Rice and E. F. C. Byrd, J. Comput. Chem., 2013, 34 (25), 2146-2151; b) M. Pospisil, P. Vavra, M. C. Concha, J. S. Murray and P. Politzer, Journal of Molecular Modeling, 2010, $16(5), 895-901$. 